\title{
EDITORIAL
}

\section{Donor blood remains a source of heavy metal exposure}

\author{
Pediatric Research (2019) 85:4-5; https://doi.org/10.1038/s41390- \\ 018-0177-7
}

To the Editor. Heavy metal (i.e., lead, mercury, and cadmium) exposure has deleterious and irreversible effects on the developing brain. ${ }^{1}$ In 2000, Bearer et al. first illustrated that blood transfusions place the smallest and most vulnerable populationextremely low birth weight (ELBW) infants-at risk of lead exposure. ${ }^{2}$ Several studies thereafter have also shown concerning levels of lead in donor blood given to neonates (Supplemental Table S1). Nearly two decades later, there is still no universal lead screening for blood going to vulnerable populations, including ELBW infants and children receiving recurrent and/or massive blood transfusions (e.g., trauma, extracorporeal membrane oxygenation, hemoglobinopathies, chronic hemolytic anemias). We reexamined the concentrations of, and variability in, heavy metals in donor blood to determine if screening donor blood for heavy metals is still warranted.

Lead, mercury, and cadmium concentrations were determined via a NexION 300D inductively coupled plasma mass spectrometer (ICP-MS) assay in 192 samples of packed red blood cell (pRBC) units selected at random from the University of Nebraska Medical Center Blood Bank, Omaha, Nebraska, United States, from July through September 2017. Samples were donated through two American Red Cross regional centers $[N=74$ (Midwest) and 118 (North Central)] across three US states. Limit of lead detection was $0.04 \mu \mathrm{g} / \mathrm{dL}$.

Lead, mercury, and cadmium concentrations in donor samples were compared to mean blood concentrations from the 2013-2014 National Health and Nutrition Examination Survey (NHANES). ${ }^{3}$ The NHANES study was designed to assess the US population generally and included measures of heavy metals. Mean lead concentrations [mean $=1.11 \pm 0.75 \mu \mathrm{g} / \mathrm{dL}$, one-sample $t$-test $t(192)=2.62, p<0.01$, test value $=0.97 \mu \mathrm{g} / \mathrm{dL}]$ and cadmium concentrations [mean $=0.49 \pm 0.46 \mu \mathrm{g} / \mathrm{L}$, one-sample $t$-test $t(192)$ $=5.94, p<0.001$, test value $=0.30 \mu \mathrm{g} / \mathrm{L}$ )] were significantly higher in donor blood (Fig. 1). Additionally, mercury in donor blood was higher than the US population at trend [mean $=1.01 \pm 1.45 \mu \mathrm{g} / \mathrm{L}$, one-sample $t$-test $\mathrm{t}(191)=1.85, p=0.066$, test value $=0.81 \mu \mathrm{g} / \mathrm{L}]$ (Fig. 1).

There were no significant differences between lead, mercury, and cadmium concentrations in the Midwest relative to the North Central donor populations $[t<1.43, p>0.15]$. However, in the North Central donor population, there were significant correlations between lead and cadmium $[r=0.42, p<0.001]$ and lead and mercury $[r=0.21, p=0.023]$. Significant correlations were not observed in the Midwest region between lead and cadmium $[r=$ $0.13, p=0.26]$ or lead and mercury $[r=0.067, p=0.57]$.

In 2003, Bearer et al. proposed an acceptable concentration of intravenous lead exposure for ELBW to be $0.09 \mu \mathrm{mol} / \mathrm{L}$ (or 1.84 $\mu \mathrm{g} / \mathrm{dL}$ ). ${ }^{4}$ This threshold was based on: the assumption of $10 \%$ lead absorption in the gastrointestinal tract; the assumption of no other lead exposure despite known lead transfer through the placenta in utero and through breastmilk); and a provisional tolerable weekly intake (PTWI) of $25 \mu \mathrm{g} / \mathrm{kg}$ based on the 1996
World Health Organization (WHO) guidelines for drinking-water quality. ${ }^{5}$ By this measure, over $14 \%$ of measured pRBC units exceeded the limit of concern for ELBW (Fig. 2). No safe level of lead has been identified. For example, the Joint (Food and Agriculture Organization and WHO) Expert Committee on Food Additives did not re-issue a PTWI, as dose-response analyses indicated that a weekly intake of the PTWI was associated with a significant decrease in intellectual functioning in children. ${ }^{5}$ Moreover, as we poorly understand how ELBW and other vulnerable populations metabolize lead, we fear even greater effects in these children. It is important to note, however, that there are still no federally mandated lead-screenings for donor blood in the United States.

While there remains no consensus safe level of lead or other heavy metal for ELBW, this research shows that heavy metal concentrations in donor blood are variable and can be greater in some regions than the national average. These data suggest that extra screening protocols for donor blood in vulnerable populations, such as ELBW infants and children receiving recurrent and/ or massive blood transfusions, may be warranted. Regional variability in the correlation of heavy metal levels, as observed in the current data, suggests that patterns of heavy metal exposure in donor populations may vary according to their geographic location. Different correlations may relate to the type of exposure to heavy metals (e.g., contaminated soil as in Environmental Protection Agency Superfund Site Omaha, Nebraska, leaded paint in older homes across the United States, ingestion through various food sources, and occupation). As such, regional, as opposed to national or international, blood screening protocols may need to be developed. Furthermore, more work is necessary to understand how high risk and vulnerable infants and children can be spared additional insults, particularly iatrogenic heavy metal exposure.

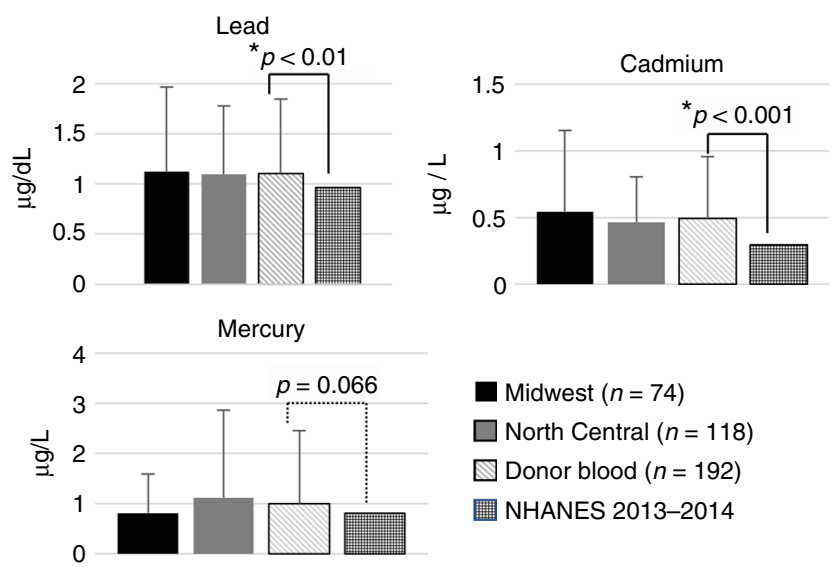

Fig. 1 Lead, cadmium, and mercury concentrations in donor samples were compared to mean blood concentrations from the 2013-2014 National Health and Nutrition Examination Survey 


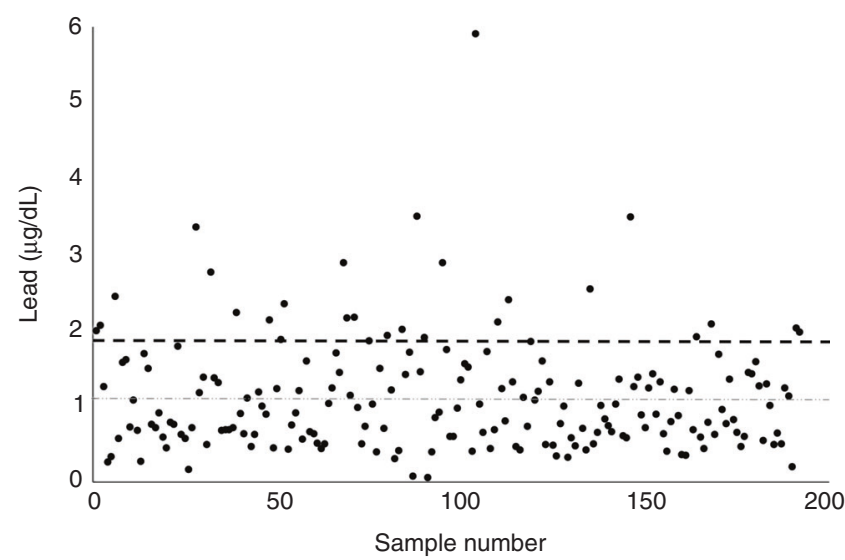

Fig. 2 Lead concentration per packed red blood cell sample with superimposed lines denoting the mean lead concentration (gray dashed line) and lead concentration of concern for extremely low birth weight infants proposed by Bearer et al. ${ }^{2}$ (bold dashed line)

\section{ACKNOWLEDGEMENTS}

We received Grant funding from the American Academy of Pediatrics Resident Research Grant and the Department of Pediatrics at the University of Nebraska Medical Center/Children's Hospital \& Medical Center.

\section{ADDITIONAL INFORMATION}

The online version of this article (https://doi.org/10.1038/s41390-018-0177-7) contains supplementary material, which is available to authorized users.
Competing interests: The authors declare no competing interests.

Publisher's note: Springer Nature remains neutral with regard to jurisdictional claims in published maps and institutional affiliations.

Kimberly M. R. White ${ }^{1}$, Ann L. Anderson Berry ${ }^{1}$, Stuart F. White ${ }^{2}$, David Moran ${ }^{3}$, Elizabeth Lyden ${ }^{4}$ and Cynthia F. Bearer ${ }^{5}$ ${ }^{1}$ Department of Pediatrics, University of Nebraska Medical Center, Omaha, NE, USA; ${ }^{2}$ Center for Neurobehavioral Research, Boys Town National Research Hospital, Boys Town, NE, USA; ${ }^{3}$ Nebraska Public Health Laboratory, University of Nebraska Medical Center, Omaha,

$N E$, USA; ${ }^{4}$ Department of Biostatistics, College of Public Health, University of Nebraska Medical Center, Omaha, NE, USA and ${ }^{5}$ Department of Pediatrics, University of Maryland School of Medicine, Baltimore, MD, USA

Correspondence: Kimberly M. R. White (kim.white@unmc.edu)

\section{REFERENCES}

1. Bellinger, D., Leviton, A., Waternaux, C., Needleman, H. \& Rabinowitz, M. Longitudinal analyses of prenatal and postnatal lead exposure and early cognitive development. N. Engl. J. Med. 316, 1037-1043 (1987).

2. Bearer, C. F., O'Riordan, M. A. \& Powers, R. Lead exposure from blood transfusion to premature infants. Pediatrics 137, 549-554 (2000).

3. Centers for Disease Control. Fourth National Report on Human Exposure to Environmental Chemical, Updated Tables, January 2017, Volume One. Available from https://www.cdc.gov/exposurereport/index.html.

4. Bearer, C. F., Linsalata, N., Yomtovian, R., Walsh, M. \& Singer, L. Blood transfusions: a hidden source of lead exposure. Lancet 363, 332 (2003).

5. World Health Organization. Guidelines for Drinking-water Quality. 4th edn. Available from: http://apps.who.int/iris/bitstream/handle/10665/44584/9789241548151_eng.pdf; jsessionid $=9 F 3966$ A1DB1BDAC86425C5992F674114? sequence $=1$ (2011). 